\title{
Metronomic Chemotherapy for Metastatic Breast Cancer - a Systematic Review of the Literature
}

\section{Die metronomische Chemotherapie bei der Behandlung von metastasierendem Mammakarzinom - eine systematische Literaturrecherche}

Authors

Affiliations
M. Banys-Paluchowski ${ }^{2}$, F. Schütz ${ }^{3}$, E. Ruckhäberle ${ }^{1}$, N. Krawczyk ${ }^{1}$, T. Fehm ${ }^{1}$

${ }^{1}$ Department of Gynecology and Obstetrics, Heinrich-Heine University Düsseldorf, Düsseldorf

${ }^{2}$ Department of Gynecology and Obstetrics, Marienkrankenhaus Hamburg, Hamburg

${ }^{3}$ Department of Gynecology and Obstetrics, Heidelberg University Hospital, University of Heidelberg, Heidelberg

\section{Key words}

- breast cancer

- metronomic chemotherapy

- survival

- toxicity

- systematic review

\section{Schlüsselwörter}

- Brustkrebs

- metronomische

Chemotherapie

- Überleben

- Toxizität

- systematische Recherche

\section{Abstract \\ $\nabla$}

Conventional chemotherapy is generally administered in high doses followed by a treatment-free period to give the body needful time to recover. This "maximum tolerated dose" approach results in high response rates. However, long periods between therapy cycles can lead to development of resistance mechanisms and consequently disease progression. One of the most interesting alternative strategies is metronomic chemotherapy. This concept relies on the continuous administration of chemotherapy at low doses and aims at targeting endothelial cells in the tumor bed as well. Recently, metronomic chemotherapy has been incorporated into the recommendations issued by the German AGO expert panel (www.ago-online. de). A systematic review of PubMed/Medline, ClinicalTrials.gov, the European Clinical Trials Database (EudraCT) and the Cochrane Database was conducted. In the present review, we discuss the current evidence on metronomic chemotherapy in metastatic breast cancer.

\section{Zusammenfassung \\ $\nabla$}

Die herkömmliche Chemotherapie wird üblicherweise in hohen Dosen verabreicht, gefolgt von einer behandlungsfreien Periode, die dem Körper die nötige Erholungszeit gibt. Dieser Ansatz, bei welchem dem Patienten die „maximal verträgliche Dosis“ verabreicht wird, ist mit hohen Ansprechraten verbunden. Die langen Abstände zwischen den einzelnen Therapiezyklen können jedoch die Entwicklung von Resistenzmechanismen begünstigen und folglich zu einem Fortschreiten der Erkrankung führen. Die metronomische Chemotherapie stellt daher eine interessante Alternative dar. Das Konzept beruht auf der kontinuierlichen Verabreichung eines niedrig dosierten Chemotherapeutikums und zielt auch auf die Endothelzellen im Tumorbett ab. Vor Kurzem wurde die metronomische Chemotherapie in die vom Expertengremium der AGO herausgegebenen Empfehlungen aufgenommen (www.ago-online.de). Es wurde eine systematische Recherche in PubMed/Medline, ClinicalTrials.gov, European Clinical Trials Database (EudraCT) sowie Cochrane Database durchgeführt. In der hier vorgestellten Literaturübersicht werden die aktuellen Erkenntnisse über die metronomische Chemotherapie bei der Behandlung von metastasierendem Brustkrebs diskutiert. $\begin{array}{ll}\text { received } & 25.2 .2016 \\ \text { revised } & 3.4 .2016 \\ \text { accepted } & 3.4 .2016\end{array}$

\section{Bibliography}

DOI http://dx.doi.org/

10.1055/s-0042-105871

Geburtsh Frauenheilk 2016; 76:

525-534 @ Georg Thieme

Verlag KG Stuttgart · New York . ISSN 0016-5751

\section{Correspondence}

Dr. med. Malgorzata

Banys-Paluchowski

Marienkrankenhaus Hamburg

Alfredstraße 9

22087 Hamburg

maggybanys@yahoo.de

\begin{tabular}{llll}
\hline \multicolumn{2}{l}{ Abbreviations } & MTD & maximum tolerated dose \\
$\nabla$ & & MTX & methotrexate \\
AE & adverse event & NPLD & non-pegylated liposomal doxorubicin \\
BC & breast cancer & ORR & overall response rate \\
BEV & bevacizumab & OS & overall survival \\
CAPE & capecitabine & PFS & progression-free survival \\
CBR & clinical benefit rate & PLD & pegylated liposomal doxorubicin \\
CEC & circulating endothelial cell & PR & partial response \\
CR & complete response & SD & stable disease \\
CTX & cyclophosphamide & TTP & time to progression \\
MBC & metastatic breast cancer & UFT & tegafur-uracil \\
MCT & metronomic chemotherapy & VIN & vinorelbine
\end{tabular}




\section{Introduction}

$\nabla$

The schedule of conventional cytostatic treatment is based on the "maximum tolerated dose" (MTD) approach where high doses of a chemotherapeutic agent are given at 2-4 week intervals and target rapidly dividing cells. Since chemotherapy does not specifically eliminate cancer cells, but rather disrupts the process of cell division, normal non-cancerous cells that proliferate at a high rate are damaged as well, leading to typical side effects, such as hair loss, bone marrow suppression and mucositis. On the other hand, the long breaks between therapy cycles can allow tumor cells to recover and develop resistance, consequently resulting in disease progression. In the last two decades, alternative strategies have been explored in order to maximize treatment response while reducing toxicity. Most importantly, targeted therapy has become a major focus of oncological research and a number of drugs directed against tumor-associated target structures has been developed. Since their efficacy is not based on proliferation, these molecules specifically eliminate tumor cells, while leaving normal cells unaffected. Secondly, new approaches to chemotherapy itself have been proposed; among them, metronomic chemotherapy (MCT) is one of the most interesting ones [1]. MCT is based on the continuous administration of cytotoxic drugs at very low doses, thus reducing side effects and shortening the rest periods between treatments.

We performed a systematic review of published clinical studies on the use of metronomic therapy in metastatic breast cancer (BC) and searched the databases of PubMed/Medline, ClinicalTrials.gov, the European Clinical Trials Database (EudraCT) and the Cochrane Database for key terms related to metronomic chemotherapy and BC. Only articles published in English were considered. Case reports and reviews were excluded from our search. For trials with more than one publication, only the latest version was included in the analysis.

\section{The Concept of Metronomic Therapy}

$\nabla$

\section{Anti-angiogenic effect}

Tumor growth depends not only on the aggressiveness of tumor cells themselves, but on the ability of endothelial cells in the tumor bed to develop new blood vessels as well. Therefore, one of the possible targets of oncologic therapy is the tumor's vascular system. High doses of chemotherapy drugs require extended periods between treatment cycles to allow non-cancerous host cells to recover and resume their activity. During these therapy-free periods, endothelial cells in the tumor may also repair some of the damage induced by the chemotherapy and resume growth. This might contribute to the fact that tumor-associated neo-angiogenesis is not efficiently targeted by traditional chemotherapy. According to several experimental studies, low doses of cytotoxic drugs, administered without interruptions at shorter intervals, may bypass this hindrance and achieve tumor regression by elimination of endothelial cells involved in angiogenesis [2]. This continuous schedule is referred to as "metronomic" or "high time" chemotherapy [1]. In contrast to the "maximum tolerated dose" approach, the high-time chemotherapy aims at administering chemotherapeutic agents for the longest time possible at a given drug concentration ("high time for low dose"). In an animal-based study, Browder et al. showed that an "antiangiogenic" metronomic schedule of cyclophosphamide provided more sustained apoptosis of endothelial cells within the tumor bed, re- gardless of whether the tumor cells were drug resistant or not [3].

\section{Continuous cytotoxic effect}

Metronomic chemotherapy may also be seen as a variation of "dose-dense" therapy. The "maximum dose" approach generally requires breaks of two to four week duration to allow recovery from damaging side effects; reducing these interruptions is referred to as "dose density". In early breast cancer, dose-dense chemotherapy, administered at frequent intervals (e.g. weekly), has been demonstrated to improve survival [4]. The weekly schedule has proved particularly beneficial when applied to taxanes. However, one major difference between dose-dense and metronomic approach is the cumulative dose, which is significantly higher in case of dose-dense therapy.

\section{Immunomodulatory effect}

Several cytotoxic drugs are able to induce immunogenic cell death. In animal-based models, metronomic administration of cyclophosphamide was shown to selectively reduce numbers of circulating regulatory $\mathrm{T}$ cells and thus curtail their immunosuppressive potential, resulting in a better control of the disease [5]. Recent studies suggested that antitumor immune responses obtained through metronomic treatment may evoke long-term immune memory leading to a rejection of tumor re-challenge in mouse models [6]. Results from immunodepletion studies suggest that tumor regression induced by metronomic therapy is mainly driven by its effects on the CD8+ T cells rather than NK cells [6]. Interestingly, even ultra-low noncytotoxic concentrations of chemotherapeutic agents, such as doxorubicin, methotrexate or paclitaxel can exercise immunomodulatory effects and directly up-regulate the ability of dendritic cells to present antigens for Ag-specific T cells in vitro [7].

\section{Review}

In the metastatic situation, therapy is mainly aimed at improving quality of life and controlling disease symptoms. In this context, metronomic chemotherapy may offer the possibility of prolonged treatment with less side effects ("high time, low dose"). A number of "older" cytotoxic drugs, such as cyclophosphamide (CTX), methotrexate (MTX), vinorelbine (VIN) and capecitabine (CAPE), have been tested in metronomic schedules. Some of the protocols include a combination of chemotherapy with antiangiogenic or endocrine therapy. The majority of ongoing trials aims at investigating low-dose metronomic capecitabine-based treatment. Table 1 gives an overview of currently ongoing and completed clinical trials phase I-III in metastatic setting.

Most trials on metronomic schedules focus on one of three settings:

1. metronomic therapy as an alternative to "conventional" chemotherapy with a more favorable safety profile;

2. metronomic therapy as a maintenance treatment after standard chemotherapy that would prolong the efficacy of conventional cytotoxic treatment;

3. metronomic chemotherapy as a combination partner for a targeted, antiangiogenic or immunologic agent. 
Table 1 Current data and ongoing trials focusing on metronomic chemotherapy in metastatic breast cancer.

\begin{tabular}{|c|c|c|c|c|c|}
\hline $\begin{array}{l}\text { Chemother- } \\
\text { apy drug }\end{array}$ & Study & Phase & $\begin{array}{l}\text { Number of } \\
\text { Patients }\end{array}$ & Study design & Results \\
\hline \multirow[t]{7}{*}{$\begin{array}{l}\text { Capecitabine } \\
\text { (CAPE) }\end{array}$} & $\begin{array}{l}\text { Stockler et al. } \\
{[12]}\end{array}$ & III & 323 & $\begin{array}{l}\text { Standard intermittent CAPE }\left(1000 \mathrm{mg} / \mathrm{m}^{2}\right. \\
\text { bid days } 1-14 \mathrm{q} 3 \mathrm{w} \text {, dose escalation } \\
\text { to } 1250 \mathrm{mg} / \mathrm{m}^{2} \text { possible) } \\
\text { vs. } \\
\text { continuous metronomic CAPE }\left(650 \mathrm{mg} / \mathrm{m}^{2}\right. \\
\text { bid) } \\
\text { vs. } \\
\text { classical CMF (oral CTX } 100 \mathrm{mg} / \mathrm{m}^{2} \text { daily days } \\
1-14+\mathrm{MTX} 40 \mathrm{mg} / \mathrm{m}^{2}+5 \text {-FU } 600 \mathrm{mg} / \mathrm{m}^{2} \\
\text { day } 1 \text { and } 8 \mathrm{q} 4 \mathrm{w} \text { ) as first-line treatment in } \\
\text { MBC patients unsuitable for more aggressive } \\
\text { regimens }\end{array}$ & $\begin{array}{l}\text { Survival: OS significantly longer in CAPE- } \\
\text { group than CMF-arm ( } 22 \text { vs. } 18 \text { months). } \\
\text { No difference between standard and metro- } \\
\text { nomic CAPE with regard to OS/PFS } \\
\text { Toxicity: significantly more serious AEs } \\
\text { in CMF than CAPE ( } 35 \text { vs. } 21 \% \text { ). } \\
\text { Toxicity similar in both CAPE arms. }\end{array}$ \\
\hline & $\begin{array}{l}\text { Saura et al. } \\
2014[32]\end{array}$ & $1 / 11$ & 72 & $\begin{array}{l}\text { Oral neratinib } 240 \text { mg daily + CAPE } 1500 \text { mg/ } \\
\mathrm{m}^{2} \text { daily in trastuzumab-pretreated HER2- } \\
\text { positive MBC }\end{array}$ & $\begin{array}{l}\text { Survival: ORR } 64 \% \text { in patients with no prior } \\
\text { lapatinib exposure and } 57 \% \text { in patients previ- } \\
\text { ously treated with lapatinib, median PFS } 40.3 \\
\text { and } 35.9 \text { weeks, respectively } \\
\text { Toxicity: diarrhea ( } 88 \% \text { ), hand-foot } \\
\text { syndrome ( } 48 \% \text { ) }\end{array}$ \\
\hline & $\begin{array}{l}\text { Ozdemir } \\
\text { et al. } 2013 \\
{[19]}\end{array}$ & II & 64 & $\begin{array}{l}\text { CAPE }\left(1000 \mathrm{mg} / \mathrm{m}^{2} \text { days } 1-14\right)+\text { cisplatin } \\
\left(60 \mathrm{mg} / \mathrm{m}^{2}\right) \mathrm{q} 3 \mathrm{w} \text {, followed by CAPE mainte- } \\
\text { nance therapy in patients with HER2-nega- } \\
\text { tive MBC pretreated with anthracycline and } \\
\text { taxane }\end{array}$ & $\begin{array}{l}\text { Survival: median TTP } 7 \text { months, } \\
\text { median OS } 17 \text { months } \\
\text { Toxicity: The most frequent grade 3-4 } \\
\text { events were neutropenia ( } 8 \% \text { ), nausea/ } \\
\text { vomiting (8\%) and thrombocytopenia (6\%). }\end{array}$ \\
\hline & $\begin{array}{l}\text { Fedele et al. } \\
2012 \text { [44] }\end{array}$ & II & 60 & $\begin{array}{l}\text { Continuous CAPE monotherapy } 1500 \mathrm{mg} \\
\text { daily in heavily pretreated patients }\end{array}$ & $\begin{array}{l}\text { Survival: median TTP } 7 \text { months, } \\
\text { median OS } 17 \text { months } \\
\text { Toxicity: Grade 3-4 uncommon; } \\
\text { haematologic toxicity } 5 \%\end{array}$ \\
\hline & $\begin{array}{l}\text { Schwartz- } \\
\text { berg et al. } \\
2014[36]\end{array}$ & II & 41 & $\begin{array}{l}\text { Oral CAPE } 1500 \text { or } 2000 \mathrm{mg} \text { daily + } \\
\text { fulvestrant in pretreated hormone receptor } \\
\text { positive HER-negative patients }\end{array}$ & $\begin{array}{l}\text { Survival: median PFS } 15 \text { months, median TTP } \\
27 \text { months, median OS } 29 \text { months, CBR } 58 \% \\
\text { Toxicity: Hand-foot-syndrome was the most } \\
\text { common AE (grade 3: } 7 \% \text {, grade 4: } 0 \% \text { ), } \\
\text { discontinuation due to AE: } 5 \% \text {. }\end{array}$ \\
\hline & $\begin{array}{l}\text { Taguchi et al. } \\
2010[45]\end{array}$ & II & 33 & $\begin{array}{l}\text { Oral CAPE } 825 \mathrm{mg} / \mathrm{m}^{2} \text { bid days } 1-21 \mathrm{q} 4 \mathrm{w} \\
\text { as first-line chemotherapy }\end{array}$ & $\begin{array}{l}\text { Survival: response rate } 18 \% \text {, } \\
\text { SD for } \geq 6 \text { months } 24 \% \text {, median PFS } \\
6.9 \text { months, median OS } 24.8 \text { months } \\
\text { Toxicity: The only grade } 3 \text { AEs were neutro- } \\
\text { penia ( } 6 \% \text { ) and hand-foot syndrome ( } 15 \% \text { ). }\end{array}$ \\
\hline & $\begin{array}{l}\text { CAMELLIA } \\
\text { (NC- } \\
\text { T01917279) }\end{array}$ & III & Ongoing & $\begin{array}{l}\text { Maintenance therapy with oral CAPE: metro- } \\
\text { nomic schedule ( } 500 \mathrm{mg} / \mathrm{m}^{2} \text { three times } \\
\text { daily without interruptions) vs. standard } \\
\text { schedule }\left(1000 \mathrm{mg} / \mathrm{m}^{2} \text { bid days } 1-14 \mathrm{q} 3 \mathrm{w}\right) \\
\text { after first-line CAPE + docetaxel chemo- } \\
\text { therapy in HER2-negative metastatic BC }\end{array}$ & Ongoing trial; no results yet available \\
\hline \multirow[t]{2}{*}{$\begin{array}{l}\text { Capecitabine/ } \\
\text { Taxane }\end{array}$} & $\begin{array}{l}\text { KBCSG-0609 } \\
\text { trial [46] }\end{array}$ & II & 43 & $\begin{array}{l}\text { Oral CAPE }\left(828 \mathrm{mg} / \mathrm{m}^{2} \text { bid days } 1-21\right)+ \\
\text { paclitaxel }\left(80 \mathrm{mg} / \mathrm{m}^{2} \mathrm{i} . \mathrm{v} \text {. days } 1,8,15\right) \mathrm{q} 4 \mathrm{w} \\
\text { as first- or second-line chemotherapy }\end{array}$ & $\begin{array}{l}\text { Survival: ORR } 46.5 \% \text {, PFS } 8.3 \text { months, OS } \\
22.9 \text { months } \\
\text { Toxicity: the most frequent grade } 3 / 4 \text { AE } \\
\text { was neutropenia ( } 28 \% \text {, leukopenia ( } 12 \%) \text {, } \\
\text { hand-foot syndrome }(9 \% \text { ) and fatigue }(7 \%)\end{array}$ \\
\hline & $\begin{array}{l}\text { BOOG 2006- } \\
06 \text { trial [11] } \\
\text { (EUDRA-CT } \\
2006- \\
006058-83 \text { ) }\end{array}$ & $\begin{array}{l}\text { II } \\
\text { Random- } \\
\text { ized }\end{array}$ & 312 & $\begin{array}{l}6 \times \text { paclitaxel }\left(90 \mathrm{mg} / \mathrm{m}^{2}\right) \text { days } 1,8 \text {, and } 15+ \\
\text { bevacizumab }(10 \mathrm{mg} / \mathrm{kg}) \text { days } 1 \text { and } 15 \mathrm{q} 4 \mathrm{w} \text {, } \\
\text { followed by bevacizumab }(15 \mathrm{mg} / \mathrm{kg}) \mathrm{q} 3 \mathrm{w} \\
\text { vs. } \\
8 \times \text { paclitaxel }\left(90 \mathrm{mg} / \mathrm{m}^{2}\right) \text { days } 1,8+\text { bevaci- } \\
\text { zumab }(15 \mathrm{mg} / \mathrm{kg})+\text { oral CAPE }\left(825 \mathrm{mg} / \mathrm{m}^{2}\right. \\
\text { bid days } 1-14) \mathrm{q} 3 \mathrm{w} \text {, followed by bevacizu- } \\
\text { mab + CAPE q3w in HER2-negative meta- } \\
\text { static or locally recurrent BC }\end{array}$ & $\begin{array}{l}\text { Survival: PFS significantly longer in the } \\
\text { CAPE arm ( } 11.2 \text { vs. } 8.4 \text { months); higher ORR } \\
\text { ( } 69 \text { vs. } 51 \% ; p=0.01 \text { ) and longer duration of } \\
\text { response ( } 6.8 \text { vs. } 5.4 \text { months) in the } \\
\text { CAPE arm; no difference in OS ( } 24.2 \text { vs. } \\
23.1 \text { months) } \\
\text { Toxicity: increased rate of grade } 3-4 \text { AEs } \\
\text { in the CAPE arm (HFS: } 34 \text { vs. } 0 \% \text { and } \\
\text { neutropenia: } 20 \text { vs. } 12 \% \text { ) }\end{array}$ \\
\hline
\end{tabular}


Table 1 Current data and ongoing trials focusing on metronomic chemotherapy in metastatic breast cancer. (Continued)

\begin{tabular}{|c|c|c|c|c|c|}
\hline \multirow[t]{4}{*}{$\begin{array}{l}\text { Chemother- } \\
\text { apy drug }\end{array}$} & Study & Phase & $\begin{array}{l}\text { Number of } \\
\text { Patients }\end{array}$ & Study design & Results \\
\hline & $\begin{array}{l}\text { CHAT trial } \\
{[13]}\end{array}$ & $\begin{array}{l}\text { II } \\
\text { Random- } \\
\text { ized }\end{array}$ & 222 & $\begin{array}{l}\text { Trastuzumab + docetaxel }\left(100 \mathrm{mg} / \mathrm{m}^{2}\right) \mathrm{q} 3 \mathrm{w} \\
\text { vs. } \\
\text { trastuzumab + docetaxel }\left(75 \mathrm{mg} / \mathrm{m}^{2}\right)+\text { CAPE } \\
\left(950 \mathrm{mg} / \mathrm{m}^{2} \text { bid days } 1-14\right) \mathrm{q} 3 \mathrm{w} \text { as first-line } \\
\text { therapy in HER2-positive MBC }\end{array}$ & $\begin{array}{l}\text { Survival: PFS significantly longer in } \\
\text { CAPE arm ( } 17.9 \text { vs. } 12.8 \text { months); } 2 \text {-year- } \\
\text { survival higher in CAPE arm ( } 75 \text { vs. } 66 \% \text { ), } \\
\text { OS data not mature yet } \\
\text { Toxicity: higher in CAPE arm (febrile neutro- } \\
\text { penia: } 27 \text { vs. } 15 \% \text {; grade } 3 / 4 \text { neutropenia: } \\
77 \text { vs. } 54 \% \text {; grade } 3 \text { HFS: } 17 \text { vs. } 1 \% \text {; grade } 3 / 4 \\
\text { diarrhea: } 11 \text { vs. } 4 \% \text { ) }\end{array}$ \\
\hline & $\begin{array}{l}\text { Mavroudis } \\
\text { et al. } 2009 \\
{[14]}\end{array}$ & III & 272 & $\begin{array}{l}\text { Docetaxel }\left(75 \mathrm{mg} / \mathrm{m}^{2}\right)+\text { epirubicin } \\
\left(75 \mathrm{mg} / \mathrm{m}^{2}\right) \mathrm{q} 3 \mathrm{w} \\
\text { vs. } \\
\text { docetaxel }\left(75 \mathrm{mg} / \mathrm{m}^{2}\right)+\text { CAPE }\left(950 \mathrm{mg} / \mathrm{m}^{2}\right. \\
\text { bid days } 1-14) \mathrm{q} 3 \mathrm{w} \text { as first-line therapy }\end{array}$ & $\begin{array}{l}\text { Survival: similar in both arms } \\
\text { (median TTP } 11 \text { months) } \\
\text { Toxicity: more hematological toxicity } \\
\text { in epirubicin arm, more HFS in CAPE arm }\end{array}$ \\
\hline & $\begin{array}{l}\text { Young et al. } \\
2012[43]\end{array}$ & II & 47 & $\begin{array}{l}\text { Docetaxel }\left(15 \mathrm{mg} / \mathrm{m}^{2} \text { weekly }\right)+\text { oral CAPE } \\
\left(1250 \mathrm{mg} / \mathrm{m}^{2} \text { daily }\right)+\text { oral celecoxib } \\
(200 \mathrm{mg} \text { bid })\end{array}$ & Survival: CBR $42 \%$, median TTP 3.6 months \\
\hline \multirow[t]{11}{*}{$\begin{array}{l}\text { Cyclophos- } \\
\text { phamide } \\
\text { (CTX)/Metho- } \\
\text { trexate (MTX) }\end{array}$} & $\begin{array}{l}\text { Colleoni et al. } \\
2006[27]\end{array}$ & $\begin{array}{l}\text { II } \\
\text { Random- } \\
\text { ized }\end{array}$ & 171 & $\begin{array}{l}\text { Two arms: oral CTX ( } 50 \mathrm{mg} \text { daily) and MTX } \\
(5 \mathrm{mg} \text { twice-weekly) } \pm \text { thalidomide } 200 \mathrm{mg} \\
\text { daily }\end{array}$ & $\begin{array}{l}\text { Survival: addition of thalidomide did not } \\
\text { improve response rate } \\
\text { Toxicity: mild; higher neurological toxicity } \\
\text { ( } 2 \text { vs. } 60 \% \text {; }<0.0001 \text { ) and constipation } \\
\text { (8vs. } 51 \% \text {; }<0.0001 \text { ) in thalidomide arm }\end{array}$ \\
\hline & $\begin{array}{l}\text { Colleoni et al. } \\
2002[47]\end{array}$ & II & 63 & $\begin{array}{l}\text { Oral CTX ( } 50 \text { mg daily) + } \\
\text { MTX ( } 5 \text { mg twice-weekly) }\end{array}$ & $\begin{array}{l}\text { Survival: CBR } 32 \%, \text { CR } 3 \% \\
\text { Toxicity: low except for elevation of liver } \\
\text { transaminases and } 2 \% \text { grade } \geq 3 \text { leukopenia }\end{array}$ \\
\hline & $\begin{array}{l}\text { Miscoria } \\
\text { et al. } 2012 \\
{[48]}\end{array}$ & II & 62 & $\begin{array}{l}\text { Oral } C T X+M T X \text { in pretreated advanced } \\
B C \text { patients }\end{array}$ & $\begin{array}{l}\text { Survival: median OS } 7.1 \text { months, } \\
\text { median PFS } 2.6 \text { months }\end{array}$ \\
\hline & $\begin{array}{l}\text { Gebbia et al. } \\
2012[49]\end{array}$ & $\begin{array}{l}\text { Retro- } \\
\text { spective }\end{array}$ & 61 & $\begin{array}{l}\text { Oral CTX } 50 \text { mg daily } \pm \text { MTX } 2.5 \text { mg twice a } \\
\text { week as second or third line of chemotherapy } \\
\text { in endocrine therapy resistant metastatic } \\
\text { patients }\end{array}$ & $\begin{array}{l}\text { Survival: TTP } 5.2 \text { months in CTX arm, } \\
6.2 \text { months in the combination arm; } \\
\text { median OS } 12.8 \text { and } 14 \text { months, respectively } \\
\text { Toxicity: both regimens well tolerated }\end{array}$ \\
\hline & $\begin{array}{l}\text { Salem et al. } \\
2008[50]\end{array}$ & II & 42 & $\begin{array}{l}\text { Oral CTX ( } 50 \mathrm{mg} \text { daily) }+ \text { MTX ( } 5 \mathrm{mg} \\
\text { twice-weekly) in heavily pretreated patients } \\
\text { ( } \geq 2 \text { lines of prior chemotherapy) }\end{array}$ & $\begin{array}{l}\text { Survival: CBR } 31 \% \text {, PR } 17 \% \text {, CR } 0 \% \\
\text { Toxicity: mild; the most common non- } \\
\text { hematological toxicity was elevation in } \\
\text { transaminases level ( } 40 \%) \text {; the only } \\
\text { grade } 4 \text { AE was neutropenia }(2.4 \%)\end{array}$ \\
\hline & $\begin{array}{l}\text { Wong et al. } \\
2010 \text { [51] }\end{array}$ & $1 / 11$ & 41 & $\begin{array}{l}\text { Daily dalteparin and oral CTX ( } 50 \mathrm{mg} \text { daily), } \\
\text { MTX ( } 5 \mathrm{mg} \text { twice-weekly), and daily } \\
\text { prednisone ( } 5 \mathrm{mg} \text { ) }\end{array}$ & $\begin{array}{l}\text { Survival: OS } 48 \text { weeks, TTP } 10 \text { weeks } \\
\text { Toxicity: minimal, transient grade } 3 \\
\text { elevation of liver transaminases: } 27 \% \text {, } \\
\text { grade } 3 \text { vomiting: } 2 \%\end{array}$ \\
\hline & $\begin{array}{l}\text { Aurilio et al. } \\
2012[37]\end{array}$ & $\begin{array}{l}\text { Retro- } \\
\text { spective }\end{array}$ & 32 & $\begin{array}{l}\text { Oral CTX } 50 \mathrm{mg} \text { daily + MTX } 5 \mathrm{mg} \\
\text { twice-weekly + fulvestrant } 250 \mathrm{mg} \mathrm{q} 4 \mathrm{w}\end{array}$ & Survival: CBR 56\%, OS 44 months \\
\hline & $\begin{array}{l}\text { Mayer et al. } \\
2012[28]\end{array}$ & i & 23 & $\begin{array}{l}\text { Oral CTX } 50 \text { mg daily + MTX } 5 \text { mg once/ } \\
\text { twice-weekly + vandetanib daily in } \\
3 \text { dose-escalation cohorts }\end{array}$ & Survival: PR $10 \%, S D \geq 24$ weeks $15 \%$ \\
\hline & $\begin{array}{l}\text { Orlando et al. } \\
2006 \text { [31] }\end{array}$ & II & 22 & $\begin{array}{l}\text { Oral CTX } 50 \mathrm{mg} \text { daily + MTX } 5 \mathrm{mg} \\
\text { twice-weekly + trastuzumab } 6 \mathrm{mg} / \mathrm{m}^{2} \mathrm{q} 3 \mathrm{w}\end{array}$ & $\begin{array}{l}\text { Survival: median TTP } 6 \text { months, CBR } 46 \% \\
\text { Toxicity: low, } 23 \% \text { grade } \geq 2 \text { liver toxicity, } \\
14 \% \text { grade } \geq 2 \text { leukopenia }\end{array}$ \\
\hline & $\begin{array}{l}\text { Garcia-Saenz } \\
\text { et al. } 2008 \\
{[21]}\end{array}$ & II & 22 & $\begin{array}{l}\text { Oral CTX ( } 50 \mathrm{mg} \text { daily) + MTX ( } 1 \mathrm{mg} / \mathrm{kg} \text { i.v. } \\
\mathrm{q} 2 \mathrm{w})+ \text { bevacizumab } 10 \mathrm{mg} / \mathrm{kg} \text { i.v. q2w } \\
\text { in pretreated BC }\end{array}$ & $\begin{array}{l}\text { Survival: CBR } 64 \% \text {, CR } 0 \% \text {, PR } 32 \% \text {, median } \\
\text { PFS } 7.5 \text { months, median OS } 13.6 \text { months }\end{array}$ \\
\hline & $\begin{array}{l}\text { Soriano et al. } \\
2011[41]\end{array}$ & II & 21 & $\begin{array}{l}\text { Oral CTX ( } 50 \text { mg daily) + } \\
\text { MTX ( } 5 \text { mg twice-weekly) + } \\
\text { five bi-weekly vaccinations (aluminum } \\
\text { hydroxide-precipitated } 1 \text { E10 anti-idiotype } \\
\text { Mab), followed by reimmunizations q } 4 \mathrm{w} \\
\text { in pretreated BC }\end{array}$ & $\begin{array}{l}\text { Survival: median TTP } 10 \text { months, median OS } \\
13 \text { months } \\
\text { Toxicity: no grade } 4 \mathrm{AEs} \text {, one grade } 3 \mathrm{AE} \\
\text { (nausea/vomiting: } 5 \% \text { ) }\end{array}$ \\
\hline \multirow[t]{2}{*}{$\begin{array}{l}\text { Cyclophos- } \\
\text { phamide }\end{array}$} & $\begin{array}{l}\text { Licchetta } \\
\text { et al. } 2010 \\
{[38]}\end{array}$ & II & 29 & $\begin{array}{l}\text { Oral CTX ( } 50 \mathrm{mg} \text { day } 1-21 \mathrm{q} 28)+ \\
\text { fractionated megestrol acetate ( } 80 \mathrm{mg} \text { bid) } \\
\text { in pretreated postmenopausal patients }\end{array}$ & $\begin{array}{l}\text { Survival: ORR } 31 \% \text {, disease control rate } 41 \% \text {, } \\
\text { mean TTP } 7.4 \text { months, mean OS } 13.4 \text { months } \\
\text { Toxicity: mild }\end{array}$ \\
\hline & $\begin{array}{l}\text { Perroud et al. } \\
2013[52]\end{array}$ & II & 15 & $\begin{array}{l}\text { Oral CTX } 50 \text { mg daily + celecoxib } 400 \text { mg } \\
\text { daily }\end{array}$ & $\begin{array}{l}\text { Survival: overall clinical benefit rate } 47 \% \text {, } \\
\text { median TTP } 14 \text { weeks, } 1 \text {-year-OS } 47 \% \\
\text { Toxicity: low: gastric grade } 1 \text { and } \\
\text { hematological grade } 1 / 2 \text {, no grade } 3 / 4\end{array}$ \\
\hline
\end{tabular}


Table 1 Current data and ongoing trials focusing on metronomic chemotherapy in metastatic breast cancer. (Continued)

\begin{tabular}{|c|c|c|c|c|c|}
\hline $\begin{array}{l}\text { Chemother- } \\
\text { apy drug }\end{array}$ & Study & Phase & $\begin{array}{l}\text { Number of } \\
\text { Patients }\end{array}$ & Study design & Results \\
\hline $\begin{array}{l}\text { Cyclophos- } \\
\text { phamide/ } \\
\text { Thiotepa/ } \\
\text { Carboplatin }\end{array}$ & $\begin{array}{l}\text { Wang et al. } \\
2015 \text { [42] }\end{array}$ & II & 23 & $\begin{array}{l}\mathrm{CTX}\left(3 \mathrm{~g} / \mathrm{m}^{2}\right)+\text { thiotepa }\left(150 \mathrm{mg} / \mathrm{m}^{2}\right)+ \\
\text { carboplatin (AUC6) } \mathrm{q} 4 \mathrm{w} \text {, followed by } \\
\text { immunotherapy ( } 3 \text { infusions of DC-CIKs) } \\
\text { and maintenance chemotherapy with oral } \\
\text { CTX } 50 \mathrm{mg} \text { daily in triple-neg. pretreated } \\
\text { metastatic BC }\end{array}$ & $\begin{array}{l}\text { Survival: PR } 13 \% \text {, SD } 56 \% \text {, PD } 30 \% \text {, median } \\
\text { PFS } 13.5 \text { months, median OS } 15.2 \text { months } \\
\text { Toxicity: The most common serious AEs } \\
\text { were neutropenia ( } 100 \% \text { ) and anemia ( } 70 \%) \text {, } \\
\text { no treatment-related deaths. }\end{array}$ \\
\hline $\begin{array}{l}\text { Cyclophos- } \\
\text { phamide/ } \\
5 \text {-FU/ } \\
\text { Vincristine/ } \\
\text { NPLD }\end{array}$ & $\begin{array}{l}\text { Manso et al. } \\
2013 \text { [53] }\end{array}$ & $\begin{array}{l}\text { Retro- } \\
\text { spective }\end{array}$ & 84 & $\begin{array}{l}\text { Oral CTX } 50 \mathrm{mg} \text { daily + prednisone } 20 \mathrm{mg} \\
\text { daily+i. v. weekly NPLD } 30 \mathrm{mg} \text { + 5-FU } 500 \mathrm{mg} \\
\text { + vincristine } 0.25 \mathrm{mg}\end{array}$ & $\begin{array}{l}\text { Survival: median } 8.4 \text { months, median } \\
\text { OS } 21 \text { months } \\
\text { Toxicity: most common grade } 2-3 \\
\text { hematologic AE: neutropenia ( } 56 \% \text { ), } \\
\text { non-hematologic AE: asthenia ( } 71 \% \text { and } \\
\text { mucositis ( } 31 \%) \text {; asymptomatic decline } \\
\text { of the left ventricular EF in } 4 \%\end{array}$ \\
\hline \multirow[t]{5}{*}{$\begin{array}{l}\text { Cyclophos- } \\
\text { phamide/ } \\
\text { Capecitabine }\end{array}$} & $\begin{array}{l}\text { SAKK 24/09 } \\
{[15]}\end{array}$ & III & 147 & $\begin{array}{l}\text { Bevacizumab + paclitaxel } \\
\text { vs. } \\
\text { bevacizumab + metronomic oral CTX }(50 \mathrm{mg} \\
\text { daily) and CAPE }(3 \times 500 \mathrm{mg} / \mathrm{d}) \text { as first-line } \\
\text { therapy in HER2-negative advanced BC }\end{array}$ & $\begin{array}{l}\text { Survival: no significant differences between } \\
\text { treatment arms } \\
\text { Toxicity: Less hair loss in metronomic arm } \\
\text { was the only clinically and statistically } \\
\text { significant difference. }\end{array}$ \\
\hline & $\begin{array}{l}\text { Wang et al. } \\
2012[54]\end{array}$ & II & 68 & $\begin{array}{l}\text { Oral metronomic CTX } 65 \mathrm{mg} / \mathrm{m}^{2} \text { days } 1-14+ \\
\text { CAPE } 1000 \mathrm{mg} / \mathrm{m}^{2} \text { bid days } 1-14 \mathrm{q} 3 \mathrm{w} \\
\text { in anthracycline/taxane-pretreated patients }\end{array}$ & $\begin{array}{l}\text { Survival: median PFS } 5.2 \text { months, } \\
\text { OS } 16.9 \text { months, overall response rate } 30 \% \\
\text { Toxicity: hand foot syndrome grade } 3: 4.4 \% \text {, } \\
\text { anorexia grade } 3 / 4: 7.5 \%\end{array}$ \\
\hline & $\begin{array}{l}\text { Yoshimoto } \\
\text { et al. } 2012 \\
{[55]}\end{array}$ & II & 51 & $\begin{array}{l}\text { Oral CAPE } 828 \mathrm{mg} / \mathrm{m}^{2} \text { bid + CTX } 33 \mathrm{mg} / \mathrm{m}^{2} \\
\text { bid days } 1-14 \mathrm{q} 3 \mathrm{w} \text { in HER2-negative patients }\end{array}$ & $\begin{array}{l}\text { Survival: median PFS } 12.3 \text { months, } 1 \text { - and } \\
\text { 2-year OS rates } 86 \text { and } 71 \% \text {, respectively } \\
\text { Toxicity: grade } 3 \text { leukopenia } 26 \% \text {, neutro- } \\
\text { penia } 16 \% \text {, no grade } 3 \text { hand-foot syndrome }\end{array}$ \\
\hline & $\begin{array}{l}\text { Dellapasqua } \\
\text { et al. } 2008 \\
{[22]}\end{array}$ & II & 46 & $\begin{array}{l}\text { Oral CAPE } 1500 \mathrm{mg} \text { daily + CTX } 50 \mathrm{mg} \text { daily + } \\
\text { i.v. bevacizumab } 10 \mathrm{mg} / \mathrm{kg} \mathrm{q2w}\end{array}$ & $\begin{array}{l}\text { Survival: overall response rate (CR+PR) } \\
\text { of } 48 \% \text {; median TTP } 42 \text { weeks } \\
\text { Toxicity: mild, grade } 3 \text { or } 4 \text { toxicity included } \\
\text { hypertension ( } 17 \%) \text {, leukopenia ( } 4 \%) \text {, } \\
\text { neutropenia ( } 4 \% \text { ), transaminitis }(4 \%) \text {, pro- } \\
\text { teinuria ( } 2 \%) \text {, nausea ( } 2 \%) \text {, vomiting ( } 2 \%)\end{array}$ \\
\hline & $\begin{array}{l}\text { Montagna } \\
\text { et al. } 2012 \\
{[24]}\end{array}$ & II & 24 & $\begin{array}{l}\text { Oral CAPE } 1500 \mathrm{mg} \text { daily + CTX } 50 \mathrm{mg} \text { daily + } \\
\text { i.v. bevacizumab } 15 \mathrm{mg} / \mathrm{kg} \text { q3w + erlotinib } \\
100 \mathrm{mg} \text { daily }\end{array}$ & $\begin{array}{l}\text { Survival: CR } 4 \%, 58 \% \text { PR, overall clinical } \\
\text { benefit rate } 75 \% \text {, median TTP } 43 \text { weeks } \\
\text { Toxicity: grade } 3 \text { AEs: diarrhea ( } 4 \% \text { ), } \\
\text { thrombosis ( } 4 \% \text { ), hypertension ( } 8 \% \text { ) }\end{array}$ \\
\hline \multirow[t]{3}{*}{$\begin{array}{l}\text { Cyclophos- } \\
\text { phamide/ } \\
\text { Capecitabine/ } \\
\text { Vinorelbine }\end{array}$} & $\begin{array}{l}\text { VEX trial } \\
\text { (EUDRA-CT } \\
2010- \\
024266-21)\end{array}$ & II & Ongoing & Oral CTX + CAPE + VIN & Ongoing trial; no results yet available \\
\hline & $\begin{array}{l}\text { Saridaki et al. } \\
2012[56]\end{array}$ & I & 36 & $\begin{array}{l}\text { Escalated doses of oral VIN (starting dose } \\
30 \mathrm{mg} \text { ) every other day + capecitabine } \\
\text { (starting dose } 800 \mathrm{mg} / \mathrm{m}^{2} \text { bid) days } 1-14 \\
\text { q3w }\end{array}$ & $\begin{array}{l}\text { Survival: CR } 5.5 \% \text {, PR } 28 \% \\
\text { Toxicity: Main AEs were grade 2-3 neutro- } \\
\text { penia ( } 17 \%), \text { grade } 2-3 \text { anemia ( } 16 \% \text { ), grade } \\
2-4 \text { fatigue ( } 28 \%) \text {, grade } 2-3 \text { nausea/ } \\
\text { vomiting ( } 11 \%) \text {, and grade } 3-4 \text { diarrhea } \\
(8 \%) \text {, no treatment-related deaths; } \\
\text { the recommended MTD doses were VIN } \\
60 \mathrm{mg} \text { and capecitabine } 1250 \mathrm{mg} / \mathrm{m}^{2} \text {. }\end{array}$ \\
\hline & $\begin{array}{l}\text { VICTOR-1 } \\
\text { trial [57] }\end{array}$ & $|-| \mid$ & $\begin{array}{l}12 \\
\text { (phase I), } \\
22 \\
\text { (phase II) }\end{array}$ & $\begin{array}{l}\text { Oral CAPE } 1500 \mathrm{mg} \text { daily + VIN 20-40 mg } \\
\text { thrice a week }\end{array}$ & $\begin{array}{l}\text { Survival: CBR } 58 \% \\
\text { Toxicity: The maximum tolerated dose of } \\
\text { VIN in phase I was } 40 \text { mg thrice a week; grade } \\
3-4 \text { toxicity in } 6 \% \text { of patients (mostly hema- } \\
\text { tological with spontaneous recovery, one } \\
\text { case of grade } 3 \text { neuropathy and one case of } \\
\text { grade } 3 \text { hand-foot-syndrome). }\end{array}$ \\
\hline \multirow[t]{2}{*}{ Vinorelbine } & $\begin{array}{l}\text { Addeo et al. } \\
2010[58]\end{array}$ & II & 34 & $\begin{array}{l}\text { Oral VIN }\left(70 \mathrm{mg} / \mathrm{m}^{2}\right) \text { days } 1,3,5 \text {, for } 3 \text { weeks } \\
\text { on and } 1 \text { week off, q4w, for a maximum } \\
\text { of } 12 \text { cycles as first-line therapy in elderly } \\
\text { patients }\end{array}$ & $\begin{array}{l}\text { Survival: PFS } 8 \text { months, OS } 16 \text { months, } \\
6 \% C R, 32 \% \text { PR }\end{array}$ \\
\hline & $\begin{array}{l}\text { De luliis et al. } \\
2015 \text { [59] }\end{array}$ & II & 32 & $\begin{array}{l}\text { Oral VIN } 30 \text { mg one day on and one day off } \\
\text { without interruptions until progression } \\
\text { or unacceptable toxicity in elderly patients }\end{array}$ & $\begin{array}{l}\text { Survival: clinical benefit } 50 \% \\
\text { Toxicity: no grade } 3 / 4 \text { toxicities }\end{array}$ \\
\hline
\end{tabular}


Table 1 Current data and ongoing trials focusing on metronomic chemotherapy in metastatic breast cancer. (Continued)

\begin{tabular}{|c|c|c|c|c|c|}
\hline \multirow[t]{3}{*}{$\begin{array}{l}\text { Chemother- } \\
\text { apy drug }\end{array}$} & Study & Phase & $\begin{array}{l}\text { Number of } \\
\text { Patients }\end{array}$ & Study design & Results \\
\hline & $\begin{array}{l}\text { Saloustros } \\
\text { et al. } 2011 \\
{[25]}\end{array}$ & ॥ & 13 & $\begin{array}{l}\text { Oral VIN ( } 50 \mathrm{mg} \text { thrice weekly) + } \\
\text { bevacizumab }(10 \mathrm{mg} / \mathrm{kg}) \text { biweekly } \\
\text { in pretreated patients }\end{array}$ & $\begin{array}{l}\text { Survival: PR } 8 \%, \text { SD } 46 \% \text {, the study was } \\
\text { closed prematurely due to lack of efficacy }\end{array}$ \\
\hline & $\begin{array}{l}\text { Tempo } \\
\text { Breast-1 trial } \\
\text { (EudraCT } \\
\text { 2014- } \\
003860-19 \text { ) } \\
\text { [60] }\end{array}$ & $\begin{array}{l}\text { II } \\
\text { Random- } \\
\text { ized }\end{array}$ & Ongoing & $\begin{array}{l}\text { Oral metronomic VIN ( } 50 \mathrm{mg} \text { thrice weekly) } \\
\text { vs. } \\
\text { Oral weekly VIN ( } 60 \mathrm{mg} / \mathrm{m}^{2} \text { weekly, increased } \\
\text { to } 80 \mathrm{mg} / \mathrm{m}^{2} \text { from the second cycle) } \\
\text { as first-line chemotherapy in hormone } \\
\text { receptor positive HER2-negative patients }\end{array}$ & Ongoing trial; no results yet available \\
\hline $\begin{array}{l}\text { Vinorelbine/ } \\
\text { Temozolo- } \\
\text { mide }\end{array}$ & $\begin{array}{l}\text { Addeo et al. } \\
2012 \text { [61] }\end{array}$ & II & 36 & $\begin{array}{l}\text { Oral temozolomide }\left(75 \mathrm{mg} / \mathrm{m}^{2}\right)+ \\
\text { whole-brain radiotherapy, followed by } \\
4 \text { weeks off-therapy, followed by oral VIN } \\
\left(70 \mathrm{mg} / \mathrm{m}^{2} \text { thrice weekly) for } 3 \text { weeks + }\right. \\
\text { temozolomide }\left(75 \mathrm{mg} / \mathrm{m}^{2} \text { days } 1-21 \mathrm{q} 4 \mathrm{w}\right) \\
\text { for } 12 \text { additional cycles in patients with } \\
\text { newly diagnosed brain metastasis }\end{array}$ & $\begin{array}{l}\text { Survival: CR } 8 \% \text {, PR } 44 \% \text {, median PFS } \\
8 \text { months, median OS } 11 \text { months }\end{array}$ \\
\hline $\begin{array}{l}\text { Irinotecan/ } \\
\text { Tegafur- } \\
\text { gimeracil- } \\
\text { oteracil } \\
\text { potassium }\end{array}$ & $\begin{array}{l}\text { Otsuka et al. } \\
2015 \text { [62] }\end{array}$ & II & 34 & $\begin{array}{l}\text { Irinotecan }\left(60 \mathrm{mg} / \mathrm{m}^{2} \text { on days } 1,8 \text {, and } 15\right. \\
\mathrm{q} 4 \mathrm{w}+\mathrm{TS}-180 \mathrm{mg} / \mathrm{m}^{2} \text { orally on days } 3-7 \text {, } \\
10-14 \text {, and } 17-21 \text { every } 4 \text { weeks in patients } \\
\text { with metastatic or recurrent BC }\end{array}$ & $\begin{array}{l}\text { Survival: response rate: } 47 \% \text {, median PFS } \\
14 \text { months, median OS } 26 \text { months } \\
\text { Toxicity: grade } 3 \text { or } 4 \text { : neutropenia ( } 15 \%) \text {, } \\
\text { leukopenia ( } 12 \%) \text {, diarrhea ( } 8 \% \text { ), and anemia } \\
(2 \%)\end{array}$ \\
\hline $\begin{array}{l}\text { 5-FU/ } \\
\text { Eniluracil }\end{array}$ & $\begin{array}{l}\text { Smith et al. } \\
2000[63]\end{array}$ & II & 33 & $\begin{array}{l}\text { Oral } 5 \text {-FU } 1 \mathrm{mg} / \mathrm{m}^{2} \text { bid + eniluracil } 10 \mathrm{mg} / \mathrm{m}^{2} \\
\text { bid days } 1-28, \mathrm{q} 35 \mathrm{~d} \text { as first-line chemo- } \\
\text { therapy }\end{array}$ & $\begin{array}{l}\text { Survival: partial response in } 55 \% \text { patients, } \\
\text { median response duration } 14 \text { months } \\
\text { Toxicity: Toxicity-associated delay and dose } \\
\text { reduction occurred in } 2 \text { and } 5 \% \text { of courses. }\end{array}$ \\
\hline
\end{tabular}

Abbreviations: AE: adverse event, PFS: progression-free survival, OS: overall survival, TTP: time to progression, CBR: clinical benefit rate, CR: complete response, PR: partial response, SD: stable disease, ORR: overall response rate, CTX: cyclophosphamide, MTX: methotrexate, CAPE: capecitabine, NPLD: non-pegylated liposomal doxorubicin

\section{Metronomic Therapy as an Alternative to Conventionally Scheduled Chemotherapy \\ $\nabla$}

The most extensively studied metronomic treatment in the metastatic setting is capecitabine-based therapy. In contrast to other drugs, the standard administration schedule of capecitabine (CAPE) is an oral regimen, usually consisting of two weeks of daily capecitabine followed by one week of rest. At first, this schedule seems to follow the metronomic concept that calls for regular intake of a cytotoxic agent without long treatment-free breaks; however, the daily administered dose is high and side effects comparable with other conventional chemotherapy drugs. In contrast, metronomic regimens work with lower doses of capecitabine and aim at long-term therapy with less toxicity. While the threshold between "metronomic" and "standard" in case of capecitabine schedule has not been strictly defined yet, in the present review we considered regimens consisting of daily doses of $<2000 \mathrm{mg} / \mathrm{m}^{2}$ as metronomic and $\geq 2000 \mathrm{mg} / \mathrm{m}^{2}$ as standard non-metronomic approach.

Capecitabine is most frequently used in the second or higher line of treatment [8]. According to the current ASCO guidelines, there are several effective agents appropriate for first-line chemotherapy and while the evidence is strongest for taxanes and anthracyclines, capecitabine is a valid option as well [9]. A recent metaanalysis showed improved PFS and response to treatment in patients treated with CAPE-based first-line therapy compared with CAPE-free therapy [10]. As expected, the safety profile differed between both groups with higher incidence of hematologic toxicity in the CAPE-free group and more gastrointestinal events and hand-foot syndrome under capecitabine-based treatment. Nine randomized controlled trials were included in the meta-analysis.
Of these, five investigated "standard"-dose capecitabine therapy (2000 $\mathrm{mg} / \mathrm{m}^{2} / \mathrm{d}$ days $1-14$ every three weeks in three trials, $2500 \mathrm{mg} / \mathrm{m}^{2} / \mathrm{d}$ in two trials). Patients in the remaining four trials were treated with daily doses $<2000 \mathrm{mg} / \mathrm{m}^{2}$ [11-14]. Stockler et al. assigned 323 patients with advanced breast cancer to one of three regimens: standard capecitabine $\left(1000 \mathrm{mg} / \mathrm{m}^{2}\right.$ twice daily for 14 of every 21 days), continuous metronomic capecitabine (650 mg/m² twice daily without breaks) and classical Bonadonna CMF regimen [12]. CAPE improved overall survival and was similarly active, less toxic, and more tolerable than CMF. No significant differences with respect to survival, tumor response and toxicity were observed between standard and metronomic CAPE schedules.

Other commonly administered metronomic regimens include cyclophosphamide (CTX) combined with either capecitabine or methotrexate (MXT) and vinorelbine(VIN)-based schedules. A direct comparison of these regimens with conventionally scheduled chemotherapy is problematic, since they were mostly tested in phase I/II non-randomized trials. The only phase III study was the Swiss SAKK 24/09 trial whose results were presented the ASCO Annual Meeting 2014 [15]. 147 patients with HER2-negative metastatic breast cancer were randomized to first-line therapy with bevacizumab (BEV) with either paclitaxel or daily oral metronomic capecitabine and cyclophosphamide. Survival was similar in both arms; with regard to toxicity profiles, lower incidence of alopecia in metronomic arm was the only clinically and statistically significant difference. While this trial failed to meet its primary endpoint of a reduced rate of prespecified grade 3-5 adverse events of metronomic therapy, the authors concluded that the combination could be an active, convenient treatment in metastatic breast cancer. 


\section{Metronomic Therapy as a Maintenance Treatment After Standard Chemotherapy \\ $\nabla$}

Longer duration of first-line chemotherapy was shown to prolong progression-free and overall survival in a meta-analysis [16]. In earlier studies, continuous treatment until progression improved quality of life compared to intermittent chemotherapy administrated for a prespecified number of cycles $[17,18]$. However, conventionally dosed chemotherapeutic agents, that follow the "maximum tolerated dose" approach, often require treatment interruption because of high toxicity. In this context, a switch to another, more tolerable regimen for a prolonged maintenance therapy might be an interesting option. The ongoing CAMELLIA trial aims at identifying the optimal schedule of capecitabine maintenance therapy after completion of first-line docetaxel/CAPE treatment (NCT01917279); the trial is currently recruiting. Smaller phase II studies investigated metronomic maintenance therapy after various schedules of conventional or combined chemotherapy. Ozdemir et al. treated 64 metastatic patients with cisplatin and low-dose capecitabine, followed by CAPE maintenance and reported acceptable toxicity and median overall survival of 17 months in this heavily pretreated group [19].

\section{Metronomic Chemotherapy as a Combination Partner for Antiangiogenic Agents \\ $\nabla$}

Animal-based studies showed an improvement in efficacy of continuous metronomic chemotherapy by adding an antiangiogenic drug $[2,20]$. This approach is based on the hypothesis that antivascular effects of a low-dose metronomic treatment might be enhanced through blockage of VEGF-mediates signals. Most studies investigating metronomic chemotherapy in combination with antiangiogenic agents focus on bevacizumab, the largest being the Dutch BOOG 2006-06 trial and the aforementioned SAKK 24/09 trial [11, 15,21]. In the BOOG 2006-06 trial, 312 HER2-negative patients with locally relapsed or metastatic disease were assigned to first-line therapy with paclitaxel and bevacizumab \pm oral CAPE $\left(825 \mathrm{mg} / \mathrm{m}^{2}\right.$ twice daily on days $\left.1-14\right)$, followed by maintenance treatment with bevacizumab alone or bevacizumab/capecitabine [11]. Patients receiving CAPE had significantly longer progression-free survival and better overall response rate than those in capecitabine-free arm, while overall survival remained similar in both groups.

Garcia-Saenz et al. reported on outcomes of 22 patients with pretreated metastatic $\mathrm{BC}$ who received metronomic oral cyclophosphamide, i. v. methotrexate and bevacizumab [21]. The treatment was well tolerated and yielded a clinical benefit rate of $64 \%$ and median PFS of 7.5 months. Dellapasqua combined another metronomic regimen (CTX/CAPE) with bevacizumab and reported similarly high clinical benefit rate (68\%) [22]; further, response to treatment was correlated to levels of circulating endothelial cells (CEC) before start of treatment. Patients with elevated CEC numbers achieved better response than patients with lower CEC level. Whether CECs, the assumed biomarker of vascular damage, might serve as a predictor of response to antiangiogenic therapy remains to be clarified in future studies [23]. An attempt to enhance the efficacy of the triple-therapy with CTX/CAPE/BEV was undertaken in a subsequent phase II trial by Montagna et al. [24]. 24 patients with metastatic HER2-negative BC with low or negative expression of hormone receptors were treated with a combi- nation of metronomic chemotherapy, bevacizumab and an EGFRinhibitor erlotinib; the overall clinical benefit rate was $75 \%$ and median time to progression 43 weeks. In contrast, discouraging results were provided by a phase II trial investigating the combination of metronomic VIN and BEV [25]. Among 13 patients included, only one achieved partial response and the trial was closed prematurely due to lack of efficacy. Another drug with antiangiogenic properties is thalidomide; besides immunomodulating activity, thalidomide was shown to inhibit VEGF-induced angiogenesis in animal models [26]. Colleoni et al. aimed at testing this hypothesis and treated 171 patients with a metronomic regimen of CTX/MTX \pm oral thalidomide in a phase II randomized trial [27]. Addition of thalidomide resulted in a significantly higher toxicity but did not increase response rates. Mayer et al. investigated the efficacy of vandetanib, another drug with antiangiogenic properties, in combination with metronomic chemotherapy in a small phase I trial [28]. Vandetanib is an oral inhibitor of VEGF-receptor and has been approved for treatment of medullary thyroid carcinoma. 23 patients with metastatic breast cancer received metronomic CTX/MTX chemotherapy and vandetanib in 3 dose-escalation cohorts; the clinical benefit rate was 25\% [28].

\section{Metronomic Chemotherapy as a Combination Partner for Targeted Therapy}

Patients with HER2-overexpressing metastatic disease benefit from HER2-targeted agents that are usually combined, at least initially, with a chemotherapy. Current standard of care for firstline therapy of HER2-positive MBC is conventionally dosed docetaxel with dual antibody blockade trastuzumab/pertuzumab [29]. Another anti-HER drug, T-DM1, is becoming the standard for second-line treatment at progression [30]. HER2-directed agents administered with metronomic therapy have been studied in phase II and III trials and remain valid options for later-line treatment. The combination of trastuzumab with dual metronomic regimen, CTX/MTX, has been evaluated in a phase II trial [31]. Besides already approved anti-HER2 agents, such as trastuzumab and lapatinib, the possibility of combining metronomic chemotherapy with novel targeted drugs has been explored. In a phase I/II dose-escalation trial, 72 trastuzumab-pretreated patients received metronomic capecitabine and neratinib, a dual inhibitor of the HER2 and EGFR kinases [32]. This treatment resulted in a median PFS of 40 weeks in patients with no prior lapatinib exposure and 36 weeks in patients previously treated with lapatinib.

\section{Metronomic Chemotherapy as a Combination Partner for Endocrine Therapy \\ $\nabla$}

The optimal sequence of chemotherapy and endocrine treatment has not been fully clarified and while some studies showed a slight significant improvement in response rates of concurrent chemo-endocrine therapy, others reported higher incidence of adverse events in case of simultaneous treatment [33,34]. In animal models, combined chemo-endocrine therapy showed the supra-additive antitumor activity compared to either monotherapy [35]. In clinical practice, conventionally scheduled, "maximum tolerated dose" cytotoxic treatment is generally administered without simultaneous anti-hormonal agents. In contrast, low-dose continuous chemotherapy may be given for prolonged 
periods without causing serious side effects and several authors explored the possibility of combining metronomic chemotherapy with endocrine treatment. Two trials examined fulvestrant and one focussed on megestrol acetate. In a phase II trial, 41 patients with hormone receptor positive and HER2-negative metastatic $\mathrm{BC}$ were treated with fulvestrant and capecitabine [36]. All patients were previously treated with at least one line of endocrine therapy in the metastatic setting. Combination therapy was well tolerated and led to a median PFS of 15 months and OS of 28.6 months. In another study, simultaneous therapy with fulvestrant and another metronomic regimen (CTX/MTX) yielded clinical benefit rate of 56\% [37]. Licchetta et al. reported on a combination of metronomic cyclophosphamide with megestrol acetate in 29 pretreated metastatic patients; the combination was welltolerated and active with mean time to progression of 7.4 months and mean OS of 13.4 months [38].

\section{Metronomic Chemotherapy as a Combination Partner for Immunomodulatory Therapy \\ $\nabla$}

In the last two decades, the role of immunomodulating agents in oncological therapy has gained considerable interest. This approach is based on the hypothesis that immune microenvironment of the tumor can be altered and become thus more hostile to cancer cells [39]. Cyclophosphamide is a strong inhibitor of FoxP3+ regulatory T-cells, leading to re-activation of tumorantigen-specific immune-reactions by T- as well as B-cells. In a small phase II study 12 metastatic BC patients were treated with cyclophosphamide $50 \mathrm{mg}$ per day. Treg level dropped down by $40 \%$ while T-effector cell level increased. Patients that responded to the immunomodulatory treatment showed a prolonged overall survival [40].

One of the currently evaluated options are vaccines designed to stimulate specific immunity to cancer antigens; several clinical trials testing therapeutic vaccines in metastatic breast cancer are ongoing. Whether the efficacy of vaccination can be increased by simultaneous low-dose chemotherapy remains to be clarified. Soriano et al. treated 21 patients with progression of metastatic disease with dual metronomic chemotherapy (CTX/MTX) combined with subcutaneous injections of 1E10 anti-idiotype vaccine, followed by monthly re-immunizations and reported a median time to progression of 9.8 months and OS of 12.9 months [41]. Wang et al. focussed on young patients with aggressive triple-negative disease who were previously treated with anthracyclines and taxanes; after salvage poly-chemotherapy with cyclophosphamide/thiotepa/carboplatin 23 patients received infusions with DC/CIKs (dendritic cells-activated cytokine-induced killer cells), followed by maintenance metronomic chemotherapy with oral cyclophosphamide [42]. The median PFS and OS were 13.5 and 15.2 months, respectively; a high rate of hematological toxicity was reported but there were no treatment-related deaths. Celecoxib, a non-steroidal anti-inflammatory agent with immunomodulatory properties has been tested in combination with docetaxel and metronomic CAPE as well [43].

\section{Conclusions}

\section{$\nabla$}

Metronomic chemotherapy has been proposed as an alternative to conventionally scheduled cytotoxic treatment following the "maximum tolerated dose" rule. In the metronomic concept the notion of "the higher the dose, the better" has been replaced by "high time, low dose", with the aim of administering systemic therapy continuously for as long as possible with minimal side effects. Metronomic chemotherapy has gained considerable interest in the field of pediatric oncology and various adult solid tumors. In breast cancer, a number of clinical trials investigated the efficacy and feasibility of this therapeutic approach. Metronomic chemotherapy is a valid option in metastatic setting. Its use has been incorporated into the recently updated guidelines issued by the German expert panel "AGO Breast Committee": metronomic therapy is recommended for patients with hormone receptor positive, HER2-negative metastatic breast cancer treated previously with taxanes and anthracyclines (www.ago-online.de).

\section{Conflict of Interest \\ $\nabla$}

The authors declare that there are no conflicts of interest.

\section{References}

1 Romiti A, Cox MC, Sarcina I et al. Metronomic chemotherapy for cancer treatment: a decade of clinical studies. Cancer Chemother Pharmacol 2013; 72: 13-33

2 Klement G, Baruchel S, Rak J et al. Continuous low-dose therapy with vinblastine and VEGF receptor-2 antibody induces sustained tumor regression without overt toxicity. J Clin Invest 2000; 105: R15-R24

3 Browder T, Butterfield CE, Kraling BM et al. Antiangiogenic scheduling of chemotherapy improves efficacy against experimental drug-resistant cancer. Cancer Res 2000; 60: 1878-1886

4 Sparano JA, Wang M, Martino S et al. Weekly paclitaxel in the adjuvant treatment of breast cancer. N Engl J Med 2008; 358: 1663-1671

5 Ghiringhelli F, Menard C, Puig PE et al. Metronomic cyclophosphamide regimen selectively depletes $C D 4+C D 25+$ regulatory $T$ cells and restores $\mathrm{T}$ and NK effector functions in end stage cancer patients. Cancer Immunol Immunother 2007; 56: 641-648

$6 \mathrm{Wu}$, Waxman DJ. Metronomic cyclophosphamide eradicates large implanted GL261 gliomas by activating antitumor Cd8 T-cell responses and immune memory. Oncoimmunology 2015; 4: e1005521

7 Shurin GV, Tourkova IL, Kaneno $R$ et al. Chemotherapeutic agents in noncytotoxic concentrations increase antigen presentation by dendritic cells via an IL-12-dependent mechanism. J Immunol 2009; 183: 137-144

8 Blum JL, Barrios CH, Feldman N et al. Pooled analysis of individual patient data from capecitabine monotherapy clinical trials in locally advanced or metastatic breast cancer. Breast Cancer Res Treat 2012; 136: 777-788

9 Partridge AH, Rumble RB, Carey LA et al. Chemotherapy and targeted therapy for women with human epidermal growth factor receptor 2negative (or unknown) advanced breast cancer: American Society of Clinical Oncology Clinical Practice Guideline. J Clin Oncol 2014; 32: 3307-3329

10 Yin W, Pei G, Liu G et al. Efficacy and safety of capecitabine-based firstline chemotherapy in advanced or metastatic breast cancer: a metaanalysis of randomised controlled trials. Oncotarget 2015; 6: 3936539372

11 Lam SW, de Groot SM, Honkoop AH et al. Paclitaxel and bevacizumab with or without capecitabine as first-line treatment for HER2-negative locally recurrent or metastatic breast cancer: a multicentre, open-label, randomised phase 2 trial. Eur J Cancer 2014; 50: 3077-3088

12 Stockler MR, Harvey VJ, Francis PA et al. Capecitabine versus classical cyclophosphamide, methotrexate, and fluorouracil as first-line chemotherapy for advanced breast cancer. J Clin Oncol 2011; 29: 44984504 
13 Wardley AM, Pivot X, Morales-Vasquez F et al. Randomized phase II trial of first-line trastuzumab plus docetaxel and capecitabine compared with trastuzumab plus docetaxel in HER2-positive metastatic breast cancer. J Clin Oncol 2010; 28: 976-983

14 Mavroudis D, Papakotoulas P, Ardavanis A et al. Randomized phase III trial comparing docetaxel plus epirubicin versus docetaxel plus capecitabine as first-line treatment in women with advanced breast cancer. Ann Oncol 2010; 21: 48-54

15 Rochlitz C, Moos R, Biger M et al. SAKK 24/09: Safety and tolerability of bevacizumab plus paclitaxel versus bevacizumab plus metronomic cyclophosphamide and capecitabine as first-line therapy in patients with HER2-negative advanced stage breast cancer-A multicenter, randomized phase III trial. J Clin Oncol 2014; 32 (Suppl.): 5s (Abstr. 518)

16 Gennari A, Stockler M, Puntoni M et al. Duration of chemotherapy for metastatic breast cancer: a systematic review and meta-analysis of randomized clinical trials. J Clin Oncol 2011; 29: 2144-2149

17 Gennari A, D'Amico M, Corradengo D. Extending the duration of firstline chemotherapy in metastatic breast cancer: a perspective review. Ther Adv Med Oncol 2011; 3: 229-232

18 Coates A, Gebski V, Bishop JF et al. Improving the quality of life during chemotherapy for advanced breast cancer. A comparison of intermittent and continuous treatment strategies. N Engl J Med 1987; 317: $1490-1495$

19 Ozdemir N, Aksoy S, Sendur MA et al. Capecitabine/cisplatin doublet in anthracycline and taxane pretreated and HER-2 negative metastatic breast carcinoma patients. J BUON 2013; 18: 831-837

20 Bello L, Carrabba G, Giussani C et al. Low-dose chemotherapy combined with an antiangiogenic drug reduces human glioma growth in vivo. Cancer Res 2001; 61: 7501-7506

21 Garcia-Saenz JA, Martin M, Calles A et al. Bevacizumab in combination with metronomic chemotherapy in patients with anthracycline- and taxane-refractory breast cancer. J Chemother 2008; 20: 632-639

22 Dellapasqua S, Bertolini F, Bagnardi V et al. Metronomic cyclophosphamide and capecitabine combined with bevacizumab in advanced breast cancer. J Clin Oncol 2008; 26: 4899-4905

23 Goon PK, Lip GY, Boos CJ et al. Circulating endothelial cells, endothelial progenitor cells, and endothelial microparticles in cancer. Neoplasia 2006; 8: 79-88

24 Montagna E, Cancello G, Bagnardi $V$ et al. Metronomic chemotherapy combined with bevacizumab and erlotinib in patients with metastatic HER2-negative breast cancer: clinical and biological activity. Clin Breast Cancer 2012; 12: 207-214

25 Saloustros E, Kalbakis K, Vardakis $N$ et al. Metronomic vinorelbine plus bevacizumab as salvage therapy for patients with metastatic breast cancer. J BUON 2011; 16: 215-218

26 D'Amato RJ, Loughnan MS, Flynn E et al. Thalidomide is an inhibitor of angiogenesis. Proc Natl Acad Sci U S A 1994; 91: 4082-4085

27 Colleoni M, Orlando L, Sanna G et al. Metronomic low-dose oral cyclophosphamide and methotrexate plus or minus thalidomide in metastatic breast cancer: antitumor activity and biological effects. Ann Oncol 2006; 17: 232-238

28 Mayer EL, Isakoff SJ, Klement $\mathrm{G}$ et al. Combination antiangiogenic therapy in advanced breast cancer: a phase 1 trial of vandetanib, a VEGFR inhibitor, and metronomic chemotherapy, with correlative platelet proteomics. Breast Cancer Res Treat 2012; 136: 169-178

29 Swain SM, Baselga J, Kim SB et al. Pertuzumab, trastuzumab, and docetaxel in HER2-positive metastatic breast cancer. N Engl J Med 2015; 372: 724-734

30 Verma S, Miles D, Gianni L et al. Trastuzumab emtansine for HER2-positive advanced breast cancer. N Engl J Med 2012; 367: 1783-1791

31 Orlando L, Cardillo A, Ghisini R et al. Trastuzumab in combination with metronomic cyclophosphamide and methotrexate in patients with HER-2 positive metastatic breast cancer. BMC Cancer 2006; 6: 225

32 Saura C, Garcia-Saenz JA, Xu B et al. Safety and efficacy of neratinib in combination with capecitabine in patients with metastatic human epidermal growth factor receptor 2-positive breast cancer. J Clin Oncol 2014; 32: 3626-3633

33 Pritchard KI. Combining endocrine agents with chemotherapy: which patients and what sequence? Cancer 2008; 112: 718-722

34 Pritchard KI, Paterson AH, Paul NA et al. Increased thromboembolic complications with concurrent tamoxifen and chemotherapy in a randomized trial of adjuvant therapy for women with breast cancer. National Cancer Institute of Canada Clinical Trials Group Breast Cancer Site Group. J Clin Oncol 1996; 14: 2731-2737
35 Kataoka M, Yamaguchi Y, Moriya Y et al. Antitumor activity of chemoendocrine therapy in premenopausal and postmenopausal models with human breast cancer xenografts. Oncol Rep 2012; 27: 303-310

36 Schwartzberg LS, Wang G, Somer BG et al. Phase II trial of fulvestrant with metronomic capecitabine for postmenopausal women with hormone receptor-positive, HER2-negative metastatic breast cancer. Clin Breast Cancer 2014; 14: 13-19

37 Aurilio G, Munzone E, Botteri E et al. Oral metronomic cyclophosphamide and methotrexate plus fulvestrant in advanced breast cancer patients: a mono-institutional case-cohort report. Breast J 2012; 18: 470-474

38 Licchetta A, Correale P, Migali C et al. Oral metronomic chemo-hormonal-therapy of metastatic breast cancer with cyclophosphamide and megestrol acetate. J Chemother 2010; 22: 201-204

39 Cimino-Mathews A, Foote JB, Emens LA. Immune targeting in breast cancer. Oncology (Williston Park) 2015; 29: 375-385

$40 \mathrm{Ge}$ Y, Domschke C, Stoiber N et al. Metronomic cyclophosphamide treatment in metastasized breast cancer patients: immunological effects and clinical outcome. Cancer Immunol Immunother 2012; 61: 353362

41 Soriano JL, Batista N, Santiesteban E et al. Metronomic cyclophosphamide and methotrexate chemotherapy combined with $1 \mathrm{E} 10$ anti-idiotype vaccine in metastatic breast cancer. Int J Breast Cancer 2011; 2011: 710292

42 Wang X, Ren J, Zhang J et al. Prospective study of cyclophosphamide, thiotepa, carboplatin combined with adoptive DC-CIK followed by metronomic cyclophosphamide therapy as salvage treatment for triple negative metastatic breast cancers patients (aged < 45). Clin Transl Oncol 2015; DOI: 10.1007/s12094-015-1339-2

43 Young SD, Lafrenie RM, Clemons MJ. Phase ii trial of a metronomic schedule of docetaxel and capecitabine with concurrent celecoxib in patients with prior anthracycline exposure for metastatic breast cancer. Curr Oncol 2012; 19: e75-e83

44 Fedele P, Marino A, Orlando L et al. Efficacy and safety of low-dose metronomic chemotherapy with capecitabine in heavily pretreated patients with metastatic breast cancer. Eur J Cancer 2012; 48: 24-29

45 Taguchi T, Nakayama T, Masuda N et al. Study of low-dose capecitabine monotherapy for metastatic breast cancer. Chemotherapy 2010; 56 : 166-170

46 Taguchi T, Yamamoto D, Masuda $N$ et al. Low dose capecitabine plus weekly paclitaxel in patients with metastatic breast cancer: a multicenter phase II study KBCSG-0609. Cancer Chemother Pharmacol 2013; 71: 741-747

47 Colleoni M, Rocca A, Sandri MT et al. Low-dose oral methotrexate and cyclophosphamide in metastatic breast cancer: antitumor activity and correlation with vascular endothelial growth factor levels. Ann Oncol 2002; 13: 73-80

48 Miscoria $M$, Tonetto F, Deroma $L$ et al. Exploratory predictive and prognostic factors in advanced breast cancer treated with metronomic chemotherapy. Anticancer Drugs 2012; 23: 326-334

49 Gebbia V, Boussen H, Valerio MR. Oral metronomic cyclophosphamide with and without methotrexate as palliative treatment for patients with metastatic breast carcinoma. Anticancer Res 2012; 32: 529-536

50 Salem DA, Gado NM, Abdelaziz NN et al. Phase II trial of metronomic chemotherapy as salvage therapy for patients with metastatic breast cancer. J Egypt Natl Canc Inst 2008; 20: 134-140

51 Wong NS, Buckman RA, Clemons $M$ et al. Phase I/II trial of metronomic chemotherapy with daily dalteparin and cyclophosphamide, twiceweekly methotrexate, and daily prednisone as therapy for metastatic breast cancer using vascular endothelial growth factor and soluble vascular endothelial growth factor receptor levels as markers of response. J Clin Oncol 2010; 28: 723-730

52 Perroud HA, Rico MJ, Alasino CM et al. Safety and therapeutic effect of metronomic chemotherapy with cyclophosphamide and celecoxib in advanced breast cancer patients. Future Oncol 2013; 9: 451-462

53 Manso L, Valdiviezo N, Sepulveda J et al. Safety and efficacy of metronomic non-pegylated liposomal encapsulated doxorubicin in heavily pretreated advanced breast cancer patients. Clin Transl Oncol 2013; 15: 467-471

54 Wang Z, Lu J, Leaw S et al. An all-oral combination of metronomic cyclophosphamide plus capecitabine in patients with anthracyclineand taxane-pretreated metastatic breast cancer: a phase II study. Cancer Chemother Pharmacol 2012; 69: 515-522 
55 Yoshimoto M, Takao S, Hirata $M$ et al. Metronomic oral combination chemotherapy with capecitabine and cyclophosphamide: a phase II study in patients with HER2-negative metastatic breast cancer. Cancer Chemother Pharmacol 2012; 70: 331-338

56 Saridaki Z, Malamos N, Kourakos P et al. A phase I trial of oral metronomic vinorelbine plus capecitabine in patients with metastatic breast cancer. Cancer Chemother Pharmacol 2012; 69: 35-42

57 Cazzaniga ME, Torri V, Villa F et al. Efficacy and safety of the all-oral schedule of metronomic vinorelbine and capecitabine in locally advanced or metastatic breast cancer patients: the Phase I-II VICTOR-1 Study. Int J Breast Cancer 2014; 2014: 769790

58 Addeo R, Sgambato A, Cennamo G et al. Low-dose metronomic oral administration of vinorelbine in the first-line treatment of elderly patients with metastatic breast cancer. Clin Breast Cancer 2010; 10 : 301-306

59 De Iuliis F, Salerno G, Taglieri L et al. On and off metronomic oral vinorelbine in elderly women with advanced breast cancer. Tumori 2015; 101: 30-35
60 De la Haba J, Cazzaniga M, Freyer G et al. Randomised phase II study evaluating, as first-line chemotherapy, single-agent oral vinorelbine administered with two different schedules in patients with hormone receptor positive, HER2-negative advanced breast cancer (TempoBreast-1 trial). In: Proceedings of the Thirty-Eighth Annual CTRC-AACR San Antonio Breast Cancer Symposium 2015. Cancer Res 2016; 76

61 Addeo R, Sperlongano P, Montella L et al. Protracted low dose of oral vinorelbine and temozolomide with whole-brain radiotherapy in the treatment for breast cancer patients with brain metastases. Cancer Chemother Pharmacol 2012; 70: 603-609

62 Otsuka H, Fujii T, Toh U et al. Phase II clinical trial of metronomic chemotherapy with combined irinotecan and tegafur-gimeracil-oteracil potassium in metastatic and recurrent breast cancer. Breast Cancer 2015; 22: 335-342

63 Smith IE, Johnston SR, O'Brien ME et al. Low-dose oral fluorouracil with eniluracil as first-line chemotherapy against advanced breast cancer: a phase II study. J Clin Oncol 2000; 18: 2378-2384 